Service social

\title{
Connaître par l'action, par Yves St-Arnaud, Montréal, Presses de l'Université de Montréal, Collection Intervenir, 1992, 112 pages.
}

\section{Lisa Verreault}

Volume 41, numéro 3, 1992

Intervenir en contexte d'autorité

URI : https://id.erudit.org/iderudit/706589ar

DOI : https://doi.org/10.7202/706589ar

Aller au sommaire du numéro

Éditeur(s)

École de service social de l'Université Laval

ISSN

1708-1734 (numérique)

Découvrir la revue

Citer ce compte rendu

Verreault, L. (1992). Compte rendu de [Connaître par l'action, par Yves

St-Arnaud, Montréal, Presses de l'Université de Montréal, Collection Intervenir, 1992, 112 pages.] Service social, 41(3), 127-128. https://doi.org/10.7202/706589ar d'utilisation que vous pouvez consulter en ligne.

https://apropos.erudit.org/fr/usagers/politique-dutilisation/ 


\section{CONNAÎTRE PAR L'ACTION}

Yves St-Arnaud

Montréal, Presses de l'Université de Montréal, Collection Intervenir, 1992, 112 pages.

Cet ouvrage récent de Yves St-Arnaud nous introduit au concept de scienceaction. II s'agit d'une méthode d'évaluation simple qui permet à l'intervenant de s'autoperfectionner et de participer à l'avancement de la science de I'intervention.

À partir de son expérience professionnelle et s'inspirant des travaux de Chris Argyris et Donald A. Schön, St-Arnaud expose d'abord les éléments qui ont conditionné le développement d'une technique scientifique mieux adaptée à la réalité de l'intervention et il présente le discours soutenant celle-ci. Il consacre ainsi le premier chapitre à l'exposition des caractéristiques principales de la science-action, aidant le lecteur à cerner les particularités de ce nouveau concept en le comparant à la science appliquée et à la recherche-action. Il discute également du caractère scientifique de la scienceaction.

L'auteur poursuit cette partie en exposant les bases méthodologiques de la science-action. II présente les principes et postulats qui la soutiennent. Ceux-ci permettent de dégager les angles d'analyse scientifique de l'évaluation de l'efficacité de l'intervention, soit l'attente et la stratégie. Partant de ces deux éléments clés, St-Arnaud dévoile la technique de collecte de données. II démontre qu'à partir de cette simple démarche de réflexion dans I'action, le praticien est en mesure d'exercer un contrôle rigoureux sur son intervention en y appliquant les principes de l'activité scientifique : la réfutabilité, l'affirmation que les situations sociales obéissent à des lois, l'analyse de la causalité et l'élégance. II ajoute que la réflexion dans I'action constitue un outil de vérification écologique des théories professées ainsi qu'un instrument d'élaboration d'un modèle d'intervention adapté à la personnalité de l'intervenant.

En complémentarité avec la partie précédente, le troisième chapitre présente la science-action comme un moyen d'avancement de la science de I'intervention. L'auteur précise que non seulement la réflexion dans l'action peut aider le praticien à devenir plus efficace et à conceptualiser son propre modèle d'intervention, mais qu'elle lui permet par la suite de participer à l'avancement de la science de l'intervention par la diffusion de ses découvertes empiriques auprès de la communauté professionnelle et universitaire. Le praticien devient alors un praticien chercheur.

St-Arnaud discute finalement des conditions d'émergence de cette méthode. Il dépeint le contexte dans lequel elle doit s'insérer et propose une démarche susceptible d'en faciliter le développement à l'intérieur des établissements universitaires. II propose à cet effet cinq règles pouvant guider l'élaboration d'une structure de formation à la science-action. 
Ces règles insistent sur :

1. la formule professionnelle comme lieu de formation;

2. un programme axé sur l'intervention où s'unissent la théorie et la pratique ;

3. I'enseignement de modèles d'intervention personnalisés ;

4. I'adoption d'une pédagogie préparant à l'éducation continue ;

5. la détermination d'un type de recherche compatible avec les objectifs professionnels.

Une telle entreprise exige évidemment la participation des milieux scientifiques et professionnels. St-Arnaud soutient que l'implantation et l'utilisation élargie de la science-action dépendra, d'une part, de la volonté de dialogue entre les acteurs ainsi que de l'intérêt des praticiens à devenir des praticiens chercheurs et, d'autre part, des bases structurelles à partir desquelles chercheurs et praticiens pourront coopérer en toute équité.

La science-action valorise le praticien en mettant son originalité et son ingéniosité professionnelle au profit de la science de l'intervention. Cet aspect de la science-action suscitera sans doute des interrogations éthiques et déontologiques. Par ailleurs, I'auteur précise que la simplicité de la technique de réflexion dans l'action ne signifie pas que son exercice se réalise facilement. Il prévient également le lecteur que la méthode qu'il suggère ne garantit aucunement l'efficacité optimale ou l'infaillibilité des interventions.

En somme, la science-action offre un outil d'auto-évaluation à l'intervenant et préconise une production de connaissances axées sur l'efficacité professionnelle. Elle apporte, bien sûr, un souffle nouveau au développement de la science de l'intervention, mais elle offre aussi un regard différent sur les rôles des praticiens et des chercheurs. Elle déclenche ainsi la réflexion et le débat entourant le cloisonnement certain qui persiste entre la pratique professionnelle et l'activité scientifique.

Enrichi de mises en situation, d'une allégorie, de nombreux schémas ainsi que d'un index, ce petit livre de St-Arnaud est clair et concis. II permet au lecteur de se familiariser rapidement avec la science-action. Bien que Connaître par l'action soit d'abord destiné aux intervenants, les professeurs et les chercheurs autant que les étudiants auraient, comme moi, enrichissements à y puiser.

Lisa VERREAULT

Étudiante à la maîtrise en service social Université Laval 